\title{
ANTENATAL FETAL SURVEILLANCE IN IUGR AND ITS OUTCOME
}

Tulika Joshi, Roopam Singh, Reeta Hansda, Kusum Das
1. Junior Resident. Department of Obstetrics \& Gynaecology, Bokaro General Hospital, Bokaro, Jharkhand.
2. Senior Consultant. Department of Obstetrics \& Gynaecology, Bokaro General Hospital, Bokaro, Jharkhand.
3. HOD \& Senior Consultant. Department of Obstetrics \& Gynaecology, Bokaro General Hospital, Bokaro, Jharkhand.
4. Senior Consultant. Department of Obstetrics \& Gynaecology, Bokaro General Hospital, Bokaro, Jharkhand.

\section{CORRESPONDING AUTHOR:}

Dr Tulika Joshi,

C/o Shri Shankar Jeen,

Q no 176, st 1, Sec 9A,

BS City, Bokaro, Jharkhand.

E-mail: tulika4014@gmail.com

OBJECTIVES: To search out IUGR cases on the basis of clinical examination (symphysiofundal height) \& to confirm them with USG at 32 weeks of gestation. To validate Modified Biophysical Profile in all confirmed cases of IUGR from 32 weeks of gestation \& all cases where AFI is decreased, NST is reactive \& pregnancy is less than 36 weeks of gestation. To validate Complete Biophysical Profile \& Color Doppler in cases: When pregnancy is less than 36 weeks of gestation \& both NST \& AFI are abnormal. STUDY DESIGN: prospective study carried out over 100 confirmed IUGR cases. MATERIALS AND METHODS: The IUGR cases were identified first by screening with SFH then confirmation with fetal abdominal circumference by USG at 32 weeks. All the cases were initially monitored with MBPP and BPP and color Doppler of Umbilical and MCA were performed in cases with abnormal MBPP having a non reactive NST. RESULTS: The incidence of IUGR was $3.86 \%$ in present study. The MBPP proved to be an effective tool for initial monitoring of IUGR cases with high specificity of $70.83 \%$. The BPP and PI indices of Umbilical and middle cerebral arteries had good correlation with perinatal outcome i.e. BPP with sensitivity and PPV of $92.3 \%$ and $85.71 \%$, Umbilical artery PI with sensitivity of $69.23 \%$ \& positive predictive value of $90 \%$ and MCA PI with high specificity of $83.33 \%$ and PPV of $93.33 \%$. CONCLUSION: MBPP is an effective initial surveillance tool in IUGR cases. The Biophysical profile, umbilical artery Doppler and Middle Cerebral artery Doppler have good correlation with perinatal outcome but further guidelines are required to integrate these surveillance modalities with each other.

KEY WORDS: IUGR, MBPP, MCA PI, UA PI

INTRODUCTION: Intrauterine growth restriction is defined as inability of a fetus to achieve its genetic growth potential. Till date its etiology is obscure and despite of intense antenatal surveillance the perinatal morbidity and mortality is high. The present antennal fetal surveillance modalities consists of daily fetal movement counts, NST, Modified biophysical profile, complete biophysical profile and Doppler study of uterine and fetal vessels. An effective protocol for integration of these diagnostic tests is yet to be defined. This study aims at diagnosis of IUGR in antenatal cases and validation of MBPP, BPP and umbilical artery \& middle cerebral artery Doppler in these cases for prediction of adverse perinatal outcome.

MATERIALS AND METHODS: Present study was a prospective study over 100 confirmed IUGR cases. The cases were selected by screening antenatal case at 32 weeks of gestation by SFH ${ }^{1}$ and 
then confirmation with fetal abdominal circumference ${ }^{2}$ by USG. Cases with congenital malformation, placenta previa and multiple pregnancies were excluded. All the confirmed cases were monitored with MBPP weekly initially and MBPP was validated in all those cases. In cases with decreased AFI only, the frequency of performing MBPP was increased to twice weekly. BPP, umbilical artery Doppler ${ }^{3}$ and MCA Doppler ${ }^{4}$ were reserved for those cases which had a non reactive NST with or without decreased AFI. The indications of delivery were term pregnancy, BPP score $\leq 4$, absent or reversed flow in umbilical artery Doppler or reversal of cerebroplacental ratio. The cesarean delivery was preferred in all the cases where Bishop Score was unfavorable or severe oligohydramnios was present. Intrapartum monitoring was done by intermittent auscultation. The outcome parameters observed were APGAR $<7$ at 5 min after birth, NICU admission and perinatal death. The sensitivity specificity, positive predictive value and negative predictive value were calculated for each test.

OBSERVATIONS AND RESULTS: A total of 2587 cases attending Antenatal OPD were screened with the help of symphysiofundal height and out of 324 screen positive cases 100 cases whose fetal Abdominal circumference is below $5^{\text {th }}$ percentile $(<2 \mathrm{SD}$ ) were labeled as confirmed IUGR cases (3.86\% incidence). Out of 100 cases, 32 cases were delivered vaginally and LSCS was done in 68 of cases. More than half of babies required NICU admissions. There were 10 early neonatal death and 2 intrauterine fetal demises in this study. Out of these two cases one patient has refused termination at 36 week of gestation when absent diastolic flow was observed and presented with IUD after 4 days. The efficacy of fetal abdominal circumference in diagnosing IUGR cases was reflected by, sensitivity of $94.44 \%$, Specificity of $28.57 \%$, positive predictive value of $77.27 \%$ \& negative predictive value of $66.67 \%$. The distribution of various parameters according to outcome is shown in table $\mathbf{I}$ :

Table I: showing distribution of demographic parameters, test results and fetal variable according to outcome

\begin{tabular}{|l|l|l|l|}
\hline Characteristic & $\begin{array}{l}\text { Good perinatal } \\
\text { outcome(48) }\end{array}$ & $\begin{array}{l}\text { Adverse perinatal } \\
\text { outcome(52) }\end{array}$ & $\begin{array}{l}\text { P } \\
\text { value }\end{array}$ \\
\hline Maternal age (years) & $24.95 \pm 2.52$ & $23.65 \pm 3.57$ & 0.71 \\
\hline $\begin{array}{l}\text { MEAN ABDOMINAL CIRCUMFERENCE } \\
\text { (mm) }\end{array}$ & $242.55 \pm 4.14$ & $238.74 \pm 4.92$ & 0.43 \\
\hline No of abnormal MBPP cases N (\%) & $14(29.16 \%)$ & $30(57.69 \%)$ & - \\
\hline $\begin{array}{l}\text { BPP performed N (\%) } \\
\text { Score>6 }\end{array}$ & $6(12.5 \%)$ & $26(50 \%)$ & \\
Score =6 & 2 & 2 & \\
Score <6 & 2 & 6 & \\
\hline Umbilical artery Doppler performed & 2 & 18 & \\
PI > 2 SD above mean n/N (\%) & 6 & 26 & \\
AERDF n/N (\%) & $2 / 6(33.33 \%)$ & $20 / 26(76.92 \%)$ & \\
& $0 / 6$ & $15 / 26(57.69 \%)$ & \\
\hline MCA PI performed & 6 & 26 & 0.36 \\
MCAPI >2 SD below mean n/N (\%) & $0 / 6$ & $14 / 26(53.84 \%)$ & 0.78 \\
\hline MEAN GESTATIONAL AGE(weeks) & $37.125 \pm 0.797$ & $36.23 \pm 0.992$ & \\
\hline Mean birth weight (kg) & $2.33 \pm 0.178$ & $2.048 \pm 0.361$ & \\
\hline
\end{tabular}


Antepartum fetal surveillance with Modified Biophysical Profile was done in all these 100 cases. There were total 44 cases with abnormal MBPP, 38 had decreased AFI and 32 cases had NR NST. All the 32 cases with NR NST were further monitored with Complete Biophysical Profile, Umbilical artery Doppler and Middle cerebral artery Doppler.

The outcome parameters were APGAR scores $<7$ at 5 min after birth, NICU admission and Perinatal death. The validity of MBPP was calculated in all the 100 cases. The validity of BPP, UA Doppler and MCA Doppler is calculated in 32 cases. The results of each test is shown in table II

Table II : Validity scores of each test for combined adverse perinatal outcome

\begin{tabular}{|l|l|l|l|l|l|l|}
\hline Test & $\begin{array}{l}\text { Outcome } \\
\text { parameter }\end{array}$ & $\mathrm{N}$ & Sensitivity & Specificity & PPV & NPV \\
\hline MBPP & APO & 100 & $57.69 \%$ & $70.83 \%$ & $68.18 \%$ & $60.71 \%$ \\
\hline BPP & APO & 32 & $92.30 \%$ & $33.33 \%$ & $85.71 \%$ & $50 \%$ \\
\hline UAPI & APO & 32 & $69.23 \%$ & $66.67 \%$ & $90 \%$ & $33.33 \%$ \\
\hline MCAPI & APO & 32 & $91.67 \%$ & $16.67 \%$ & $42.30 \%$ & $75 \%$ \\
\hline
\end{tabular}

DISCUSSION: Antenatal fetal surveillance is of utmost importance in cases of IUGR where decision of time of delivery is very crucial. Incidence of IUD is high among these cases so intense fetal surveillance is helpful in decision making for time of delivery; still one has to keep in mind the cost and time expenditure associated with these procedures. In the present study diagnosing IUGR the validity of AC below $5^{\text {th }}$ percentile were sensitivity $94.44 \%$, specificity $28.57 \%$, positive predictive value of $77.27 \%$ and Negative predictive value of $66.67 \%$. On comparing the results the sensitivity and Positive predictive values are higher and specificity \& negative predictive value are lower than other studies ${ }^{5,6}$. The reason of disparity may be the difference in the cut off AC percentiles values, size of study population and the test was applied to a population already suspected to have IUGR (on the basis of SFH) in the present study.

The validity of MBPP in present study was sensitivity $57.69 \%$, specificity $70.83 \%$, positive predictive value $68.18 \%$ \& negative predictive value $60.71 \%$. The sensitivity and specificity are comparable to Bardakci et al7 (60\% \& 87.1\%). The variation in PPV and NPV was seen which can be attributed to difference in sample size and application of test in prescreened population (with SFH and AC) in the present study. The trend of results is similar to Jamal et al ${ }^{8}$ though they had found a very high validity scores.

There was good correlation of biophysical profile score with perinatal outcome reflected by sensitivity and positive predictive value of $92.3 \%$ and $85.71 \%$ respectively. The specificity and Negative predictive value were low i.e. $33.33 \%$ and $50 \%$ respectively. The sensitivity and PPV are comparable to Jamal et $\mathrm{al}^{8}(84.6 \%$ and $91.7 \%)$ but there is disparity in specificity and negative predictive value $(97.4 \%$ and $95 \%)$. Performing of BPP in a population already having one or two abnormal components (NST \& AFI) may be the reason of disparity.

There was poor correlation of Umbilical artery PI with perinatal outcome except for a high positive predictive value (sensitivity 69.23\%, specificity 66.67\%, PPV 90\% and NPV $33.33 \%$ ). The results are comparable to Dhand et al ${ }^{9}$ except for sensitivity (44\%) which is higher in the present study. The difference may be due to performing of UA Doppler in the prescreened population in the present study. Turan et al ${ }^{10}$ had performed the UA Doppler in the prescreened population and the sensitivity and specificity (65\% and $54 \%$ respectively) were comparable to the present study but the PPV and NPV differ from present study (37\% and 79\% 
respectively). The reason may be Turan et al had used UA PI $>2$ SD above mean as diagnostic criteria for IUGR and AREDF in UA as prognostic criteria whereas in present study UA PI > 2 SD above mean is prognostic criteria.

In the present study MCA PI has shown good specificity of $83.33 \%$ and positive predictive value of $93.33 \%$ which is comparable to Dhand et al ${ }^{9}$. The sensitivity $(53.84 \%)$ and negative predictive (29.41\%) value of the MCA PI of present study is lower than that of Dhand et $\mathrm{al}^{9}(94 \%$ and $65 \%)$. The difference in sensitivity and negative predictive value may be attributed to different sample sizes. Validity scores of present study are in accordance with the study done by Lakhrar et al ${ }^{11}$ but differ totally from Turan et al ${ }^{10}$ due to previously explained reasons.

There are changes observed in the antenatal fetal surveillance in IUGR cases such as; use of UA PI $>2$ SD above mean as diagnostic modality and reserving absent or reversed end diastolic flow for prognostic purpose. Recently Dhand et al stressed the importance of MCA PI in management of late onset IUGR cases and role of absent diastolic pulsation of MCA as indicator of adverse perinatal outcome.

MBPP is an effective initial surveillance tool in IUGR cases. Though it has a low sensitivity (57.69\%) but it has good specificity, PPV and NPV. By using MBPP as initial surveillance method one saves time, resources and cost of monitoring. The 'at risk' population is effectively selected and resources can be directed to more needful cases.

The Biophysical profile, umbilical artery Doppler and Middle Cerebral artery Doppler have good correlation with perinatal outcome but further guidelines are required to integrate these surveillance modalities with each other.

Each day gained in utero is boon to fetus but in IUGR cases prolongation of intrauterine life should be carefully balanced with risk of intrauterine death. The selection of appropriate time of delivery is of utmost importance in management of intrauterine growth restriction.

\section{REFERENCES:}

1. Rai L, Kurien L, Kumar P. Symphysis fundal height curve--a simple method for foetal growth assessment. J Postgrad Med 1995;41:93

2. Rashid S Q . Fetal Abdominal Circumference Growth in Bangladeshi Population. Journal of Bangladesh College of Physicians and Surgeons. 2008.26(3);124-127.

3. Chanprapaph P, Tongsong T, Wanapirak C. Umbilical Artery Doppler waveform indices in normal pregnancies. Thai Journal of Obstetrics and Gynaecology June 2000, Vol. 12, pp. $103-107$

4. Komwilaisak R, Saksiriwuttho P, Ratanasiri T, Kleebkaow P, Seejorn K.Pulsatality index of middle cerebral artery in normal fetuses. J Med Assoc Thai 2004; 87(Suppl 3): S34-7.

5. De Reu PA, Smits LJ, Oosterbaan HP, Nijhuis JG. Value of a single early third trimester fetal biometry for the prediction of birth weight deviations in a low risk population. J Perinat Med. 2008 ;36(4):324-9.

6. Kayem G, Grangé G, Bréart G, Goffinet F. Comparison of fundal height measurement and sonographically measured fetal abdominal circumference in the prediction of high and low birth weight at term. Ultrasound Obstet Gynecol. 2009 Nov ;34(5):566-71.

7. Bardakci M, Balci O, Acar A, Colakoglu MC. Comparison of modified biophysical profile and doppler ultrasound in predicting the perinatal outcome at or over 36 weeks of gestation.Gynecol Obstet Invest. 2010 ;69(4):245-50. Epub 2010 Jan 12 . 
8. Jamal A, Maroosi V, Eslamian L, Noori K. A prospective trial of the Fetal Biophysical Profile Vs Modified Biophysical profile in management of high risk pregnancies. Acta Medica Iranica 2007; 45(3): 204-208.

9. Dhand H, Kumar KH, Dave A. Middle cerebral artery Doppler indices better predictor for fetal outcome in IUGR. J Obstet Gynecol India. 2011;61:166-71.

10. Turan S, Turan OM, Berg C, Moyano D, Bhide A, Bower S, Thilaganathan B, Gembruch U, Nicolaides K, Harman C, Baschat AA. Computerized fetal heart rate analysis, Doppler ultrasound and biophysical profile score in the prediction of acid-base status of growthrestricted fetuses. Ultrasound Obstet Gynecol. 2007 Oct ;30(5):750-6.

11. Lakhrar BN, Rajgopal KV, Gourisankar PT. Doppler predictions of adverse perinatal outcome in PIH \& IUGR. Ind J Radiol Imag 2006 16:1:109-116. 